\title{
ASIC2 Subunits Target Acid-Sensing Ion Channels to the Synapse via an Association with PSD-95
}

\author{
Xiang-ming Zha, ${ }^{1,3}$ Vivian Costa, ${ }^{2,3}$ Anne Marie S. Harding, ${ }^{3}$ Leah Reznikov, ${ }^{1,3}$ Christopher J. Benson, ${ }^{2,3}$ and \\ Michael J. Welsh ${ }^{1,2,3,4}$ \\ ${ }^{1}$ Howard Hughes Medical Institute, ${ }^{2}$ Neuroscience Program, and Departments of ${ }^{3}$ Internal Medicine and ${ }^{4}$ Molecular Physiology and Biophysics, Roy J. and \\ Lucille A. Carver College of Medicine, University of Iowa, Iowa City, Iowa 52242
}

Acid-sensing ion channel-1a (ASIC1a) mediates $\mathrm{H}^{+}$-gated current to influence normal brain physiology and impact several models of disease. Although ASIC2 subunits are widely expressed in brain and modulate ASIC1a current, their function remains poorly understood. We identified ASIC2a in dendrites, dendritic spines, and brain synaptosomes. This localization largely relied on ASIC2a binding to PSD-95 and matched that of ASIC1a, which does not coimmunoprecipitate with PSD-95. We found that ASIC2 and ASIC1a associated in brain, and through its interaction with PSD-95, ASIC2 increased ASIC1a localization in dendritic spines. Consistent with earlier work showing that acidic $\mathrm{pH}$ elevated spine $\left[\mathrm{Ca}^{2+}\right]_{\mathrm{i}}$ by activating ASIC1a, loss of ASIC2 decreased the percentage of spines responding to acid. Moreover, like a reduction of ASIC1a, the number of spine synapses fell in ASIC2 ${ }^{-/-}$ neurons. These results indicate that ASIC2 facilitates ASIC1a localization and function in dendritic spines and suggest that the two subunits work in concert to regulate neuronal function.

\section{Introduction}

Acid-sensing ion channel 1a (ASIC1a) is a proton-gated cation channel expressed in the soma and the dendritic spines, the site of most excitatory synaptic transmission (Waldmann et al., 1997; Wemmie et al., 2002; Zha et al., 2006; Chai et al., 2007). Consistent with this localization, disrupting the ASIC1a gene in mice impairs hippocampal long-term potentiation (LTP) and spatial learning (Wemmie et al., 2002). ASICla is also highly expressed in the amygdala where it contributes to fear-conditioning and innate fear (Wemmie et al., 2002, 2003, 2004; Coryell et al., 2007). Consistent with a role for ASIC1a in synaptic function, we recently showed that changing ASICla levels alters acid-evoked $\mathrm{Ca}^{2+}$ transients in dendritic spines and influences the density of dendritic spines (Zha et al., 2006).

ASIC2 (including ASIC2a and -2b, alternatively spliced subunits) is widely expressed in the CNS (CNS) (Waldmann et al., 1996; Price et al., 1996; García-Añoveros et al., 1997). Yet compared with ASIC1a, we know less about its function there. Homomultimeric ASIC2a channels are much less $\mathrm{pH}$-sensitive than

\footnotetext{
Received March 16, 2009; revised April 24, 2009; accepted May 13, 2009.

C.J.B. is supported by the National Heart, Lung, and Blood Institute (NHLBI) (Grant HL076419). X.-m.Z. is a Research Specialist, L.R. is an Associate, and M.J.W. is an Investigator of the Howard Hughes Medical Institute. We thank Tami Nesselhauf and Pamela Hughes for cell culture [ In Vitro Models and Cell Culture Core partially supported by the NHLBI (Grant HL51670), the National Institute of Diabetes and Digestive and Kidney Diseases (Grant DK54759), and the Cystic Fibrosis Foundation]. We thank Karin Rod, Yu F. Li, and Sarah Malerich for technical assistance.

This article is freely available online through the J Neurosci Open Choice option.

Correspondence should be addressed to either of the following: Michael J. Welsh, Howard Hughes Medical Institute, Roy J. \& Lucille A. Carver College of Medicine, lowa City, IA 52242, E-mail: michael-welsh@uiowa.edu; or Christopher J. Benson, Roy J. \& Lucille A. Carver College, Department of Internal Medicine, E314-1 GH, lowa City, IA 52242-1009, E-mail: chris-benson@uiowa.edu.

DOI:10.1523/JNEUROSCI.1284-09.2009

Copyright $\odot 2009$ Society for Neuroscience $\quad$ 0270-6474/09/298438-09\$15.00/0
}

ASIC1a channels (Lingueglia et al., 1997; Benson et al., 2002), and ASIC2b homomultimers do not generate $\mathrm{H}^{+}$-gated currents. These data suggest that ASIC2 homomultimers do not likely mediate the response to acidic $\mathrm{pH}$ within the physiological $\mathrm{pH}$ range. However, electrophysiological data suggest that ASIC2 subunits expressed in heterologous cells form heteromultimers with ASICla to generate $\mathrm{H}^{+}$-gated currents that manifest properties different from those generated by either subunit alone (Benson et al., 2002; Berdiev et al., 2003; Askwith et al., 2004). Most hippocampal and cortical neurons manifest $\mathrm{H}^{+}$-gated currents that appear to arise from a combination of ASIC1a and ASIC2 subunits, and coexpressing ASIC2a with ASIC1a reproduced those properties (Baron et al., 2002a; Askwith et al., 2004; Gao et al., 2004). Moreover, cultured neurons from ASIC2 null mice have altered currents with properties like those of ASICla homomeric channels (Askwith et al., 2004). These results suggested that the absolute and relative amounts of ASIC2 subunits may determine the properties of $\mathrm{H}^{+}$-gated currents. However, whether ASIC2 has additional functions is unknown.

In this study, we used hippocampal slices and brain to better elucidate the role of ASIC2 in central neurons. Based on the studies described above, we hypothesized ASIC2 subunits function in concert with ASIC1a. Moreover, given our recent finding that ASIC1a has functional effects in dendritic spines, we asked whether ASIC2a plays a role at the postsynaptic sites of excitatory synapses.

\section{Materials and Methods}

Mice. Wild-type, $A S I C 2^{-/-}$, and $A S I C 1 a^{-/-}$mice were on a congenic C57BL/6 background. The congenic knock-outs were initially backcrossed 10 times to C57BL/6 background and refreshed (backcrossed to C57BL/6 wild-type) every 5-10 generations, according to The Jackson Laboratory's recommendations. Details on the generation and character- 
A
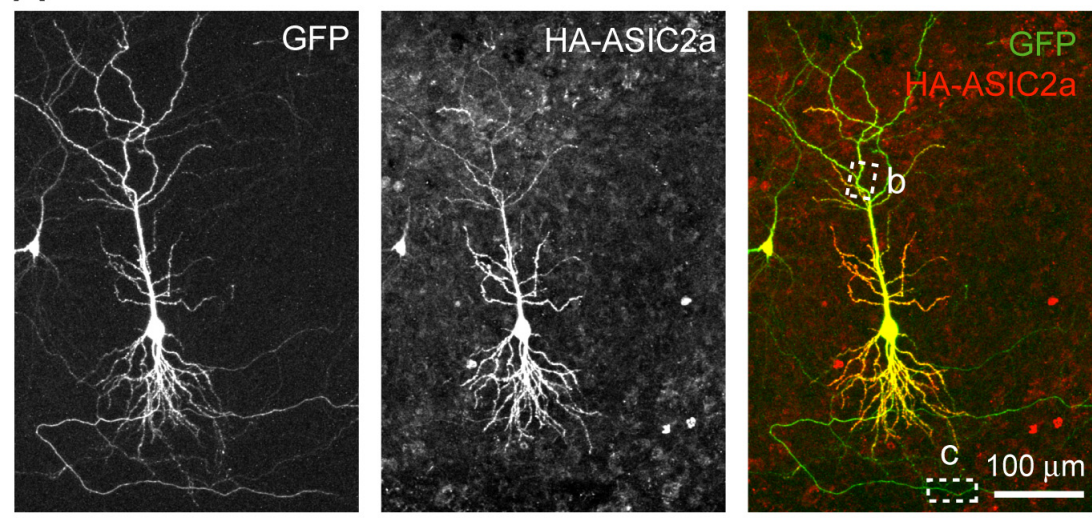

B Dendrite (region $b$ in panel $A$ )
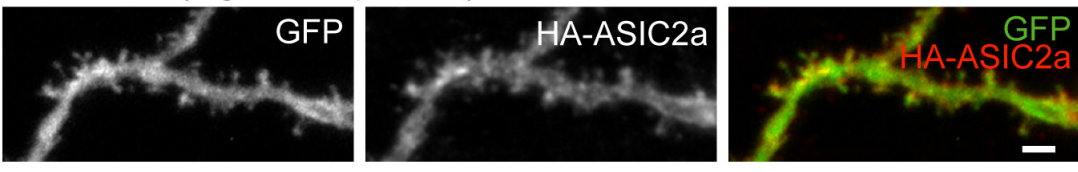

C Axon (region $b$ in panel $A$ )
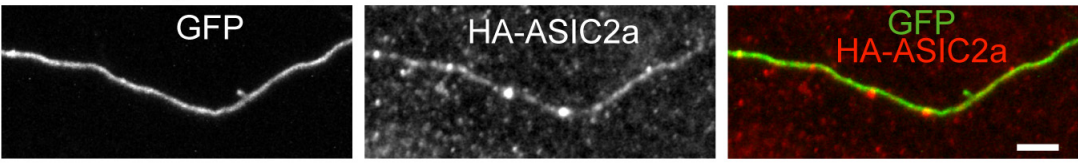

D
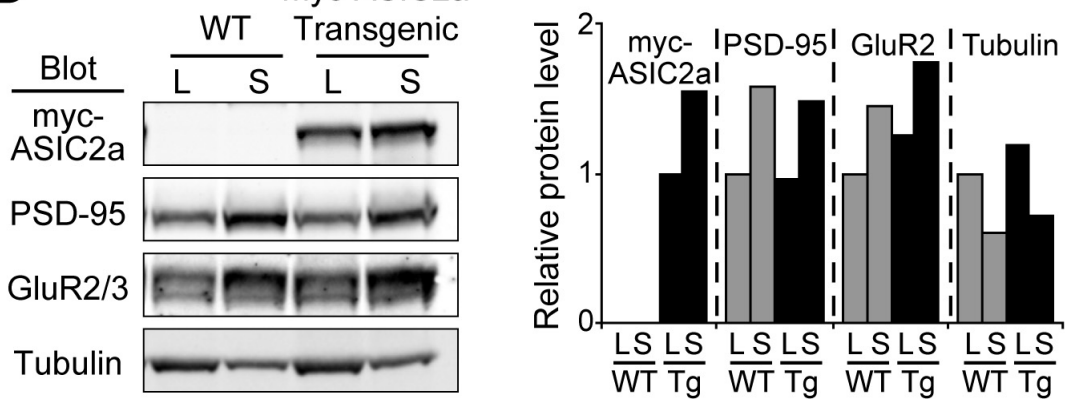

Figure 1. ASIC2a localizes to dendrites and spines in hippocampal slices. Hippocampal slices were cotransfected with HAASIC2a and GFP. A, Images showing the overall distribution of ASIC2a and GFP in a CA1 pyramidal neuron. GFP fluorescence is shown on the left, ASIC2a immunofluorescence in the middle, and merged images (GFP in green, ASIC2a in red) on the right. The images shown are representative of 4 similar experiments. $\boldsymbol{B}$, High-magnification view of a segment of an apical dendrite, illustrated by the boxed area $\boldsymbol{b}$ in $\boldsymbol{A}$. Note the presence of ASIC2a in most spines. $\boldsymbol{C}$, High-magnification view of a segment of axon, illustrated by the boxed area $c$ in $A$. Note that the selected axonal region was $\sim 600 \mu \mathrm{m}$ away from cell body, and the signal intensity was increased to show the low levels of ASIC2a in axons. D, Representative Western blots of total lysate (L) and synaptosomal fraction (S) of whole brain from wild-type and Myc-ASIC2a transgenic mice. Equal amounts (50 $\mu \mathrm{g})$ of protein were loaded per lane. Myc-ASIC2a was enriched in the synaptosomal fraction. PSD-95, GluR2/3 and tubulin served as controls. Note that whereas PSD-95 and GluR2/3 were enriched, tubulin was decreased in the synaptosomal fraction. All four antibodies were added to the same blot at the same time. Blots shown were representative of data from two transgenic mice. The right panel shows the quantification of the blot.

ization of transgenic mice carrying Myc-ASIC2a will be described elsewhere (V.C. and M.J.W., unpublished data). In these mice, Myc-ASIC2a was driven by a pan-neuronal synapsin I promoter. The expression of transgene was verified by Western blot (Fig. $1 D$ ). Animal care met National Institutes of Health standards and all procedures were approved by the University of Iowa Animal Care and Use Committee.

Constructs and reagents. PSD-95-GFP, mStrawberry, and HA-sodium/ hydrogen exchanger 1 (NHE1) were kindly provided by Drs. David Bredt, Roger Tsien, and John Orlowski, respectively. The Myc epitope in Myc-PSD-95 was deleted by site-directed mutagenesis. ASIC2a and
mStrawberry were subcloned into the eGFP-C1 vector, where eGFP was eliminated. HA-mouse ASIC1a (Zha et al., 2009) and Flag-human ASIC1a (Wemmie et al., 2004) were described earlier. HA-ASIC2a was generated by inserting one copy of an HA epitope (YPYDVPDYA) at the $\mathrm{N}$ terminus of mouse ASIC2a. The PDZbinding motif mutant of HA-ASIC2a was generated by site-directed mutagenesis (Stratagene), replacing the C-terminal 3 aa with GlyAla-Ala (ASIC2 $\mathrm{a}_{\mathrm{GAA}}$ ).

The antibodies used were: rabbit polyclonal anti-HA antibody (Abcam), rabbit anti-ASIC1 (Wemmie et al., 2002), rat monoclonal anti-HA (Roche), mouse monoclonal anti-HA and goat anti-ASIC1 (Santa Cruz Biotechnology and Abcam), mouse monoclonal anti-GFP (Clontech and Roche), rabbit polyclonal anti-GFP (Invitrogen), goat polyclonal anti-mouse HRP conjugated secondary antibody (GE Healthcare BioSciences and Millipore) and Alexa 488-, 568-, 680-, and 800-conjugated secondary antibodies (Invitrogen and LI-COR). For detecting endogenous ASIC2 on Western blot, we tried commercially available antibodies from Alomone Labs, Alpha Diagnostic, Millipore Bioscience Research Reagents, Osenses, and Santa Cruz Biotechnology. Consistent with a previous report (Ettaiche et al., 2004), we found that most ASIC2 antibodies did not recognize endogenous ASIC2 specifically. With the blotting conditions we used, only one antibody (MDEG11-A) from Alpha Diagnostic recognized a specific band at the correct size using $A S I C 2^{-/-}$brain as the negative control (see Fig. 5).

Hippocampal slice culture, transfection, and immunofluorescence. Mouse hippocampal organotypic slice culture and biolistic transfection of slice cultures were done as described previously (Zha et al., 2005, 2006). For synapse analysis, P6 mice were cultured for $6 \mathrm{~d}$ before transfection or P7 mice were cultured for $5 \mathrm{~d}$ before transfection. Slices were fixed with fixation medium (4\% paraformaldehyde in $\mathrm{HBSS}^{+/+}$ buffer containing $6 \mathrm{mg} / \mathrm{ml}$ glucose and $25 \mathrm{~mm}$ HEPES, pH 7.3) 48-52 h after transfection. Comparisons between different conditions were performed on slices dissected and cultured at the same time. Immunofluorescence was done as described earlier (Zha et al., 2006).

Cell culture and transfection. COS-7 cells were cultured as described earlier (HruskaHageman et al., 2002). Transfection was done with electroporation or Lipofectamine 2000 (Invitrogen) following the manufacturer's instructions.

Immunoprecipitation, synaptosomal fractionation and Western blot. For coimmunoprecipitation of ASIC1a and ASIC2a in vivo, whole brains of wild-type, $\mathrm{ASIC}^{-/-}$, and Myc-mASIC2a transgenic mice were homogenized in lysis buffer (1\% Triton X-100, $50 \mathrm{~mm}$ Tris pH 7.4, $150 \mathrm{~mm} \mathrm{NaCl}$ ) containing EDTA-free protease inhibitor cocktail (Roche). Lysates were cleared by centrifugation at $14,000 \mathrm{rpm}$ for $30 \mathrm{~min}$ at $4^{\circ} \mathrm{C}$ or at $200,000 \times$ $g$ for $30 \mathrm{~min}$ at $4^{\circ} \mathrm{C} .300-500 \mu \mathrm{g}$ of total protein was used per immunoprecipitation. Immunoprecipitation was performed $4 \mathrm{~h}$ to overnight at $4^{\circ} \mathrm{C}$ using anti-ASIC1 antibody (Wemmie et al., 2002), anti-Myc antibody, control IgG, or anti-GluR2/3. The complex was captured with 30 
or $60 \mu \mathrm{l}$ of $50 \%$ slurry of immobilized Protein A agarose bead (Invitrogen). Immunoprecipitates were collected by centrifugation and washed 3-4 times with TBST or IP wash buffer ( $25 \mathrm{~mm}$ Tris pH7.4, $150 \mathrm{~mm} \mathrm{NaCl}$, $0.1 \%$ Triton X-100). For coimmunoprecipitation of myc-ASIC2a and PSD-95 from brain, the wash buffer was ice-cold PBS ${ }^{+/+}$because a more stringent wash disrupted the interaction.

For COS cell immunoprecipitation, cells were washed 3 times with ice-cold PBS 24-48 h after transfection. Cells were then removed by scraping into $400 \mu \mathrm{l}$ of lysis buffer [ $25 \mathrm{~mm}$ Tris, $\mathrm{pH}$ 7.4, $1 \%$ Triton, 150 $\mathrm{mm} \mathrm{NaCl}$ with freshly added protease inhibitor cocktail (Roche)], drill homogenized or sonicated. Lysate was cleared by centrifugation for $15-20 \mathrm{~min}$ at $14,000 \mathrm{rpm}$ at $4^{\circ} \mathrm{C}$. The supernatant was then precleared with $50 \mu \mathrm{l}$ of Protein A agarose beads. Standard immunoprecipitation with anti-GFP antibody was performed overnight at $4^{\circ} \mathrm{C}$ and the complex was captured with $50 \mu \mathrm{l}$ of immobilized Protein A agarose beads. The immunoprecipitates were collected by centrifugation and washed 3-4 times with $0.1 \%$ or $1 \%$ Triton X-100 lysis buffer. When coexpressed with ASIC $2 \mathrm{a}_{\mathrm{GAA}}$, the total amount of PSD-95 protein was reduced compared with what it was when coexpressed with ASIC2a. Therefore, to start with equal amounts of PSD-95 protein, we used 50\% more lysate for the ASIC2 $\mathrm{a}_{\mathrm{GAA}}$ groups (Fig. 2).

Synaptosomes from whole brain were isolated as described (Matsuda and Cooper, 1981) using 3- to 4-week-old congenic C57BL/6 male mice. Equal amounts of protein $(25$ or $50 \mu \mathrm{g})$ were loaded per lane.

Proteins were separated on either $7.5 \%$ or $10 \%$ polyacrylamide SDS gels and transferred to PVDF (Millipore) or PROTRAN nitrocellulose membranes (PerkinElmer) after electrophoresis. Standard Western blot protocol was performed as described earlier (Zha et al., 2009). Antibody dilutions: anti-HA-HRP 1:750, anti-GFP 1:1000, anti-HA 1:1000, antitubulin 1:4000-10,000, anti-GluR2/3 1:1000, anti-PSD-95 1:500, rabbit anti-ASIC1 1:3000-5000, goat anti-ASIC1 1:1000, and rabbit antiASIC2a 1:50. Secondary antibodies used were: donkey or goat antimouse-HRP, Alexa 800 or 680-conjugated donkey or goat anti-rabbit and anti-mouse and donkey anti-goat antibodies (all used at 1: 400020,000). Chemiluminescent detection or imaging with an Odyssey imaging system (LI-COR Inc.) and quantification was performed as described earlier (Zha et al., 2009).

Confocal microscopy and cameleon imaging. Confocal and multiphoton images were captured using a two-photon microscope (Zeiss LSM 510 META) as described earlier (Zha et al., 2006). For $\left[\mathrm{Ca}^{2+}\right]_{\mathrm{i}}$ imaging, hippocampal slices were transfected with cameleon YC3.60 and FRET imaging in response to acid addition was done as described earlier (Zha et al., 2006). For synapse analysis, one segment of an apical dendrite ( $\sim 50$ $150 \mu \mathrm{m}$ away from the cell body layer) from each transfected neuron was imaged and used for analysis. The image field of view was $\sim 60-80 \mu \mathrm{m}$ and thus covered a large fraction of the medial portion of an apical dendrite. Analyses were done blinded.

Quantification of ASIC immunofluorescence, $\left[\mathrm{Ca}^{2+}\right]_{i}$ response, and synapse number. All quantifications were done with the observer blinded to the genotype and experimental conditions. ASIC1a and ASIC2a expression level was quantified in NIH ImageJ. A line was drawn across the spines and their adjacent shafts, and raw ASIC immunofluorescence intensities were measured. The maximum value was used for a given spine or shaft area. When comparing two groups cotransfected with soluble GFP, as a control for changes in volume, spine/shaft ratio of ASIC was normalized to that of GFP. For comparison purposes, the spine/shaft ASIC ratio obtained for controls was arbitrarily set to 1 .

For quantification of $\left[\mathrm{Ca}^{2+}\right]_{\mathrm{i}}$ responses, raw CFP and YFP fluorescence intensity of each spine was quantified in the imaging software (Zeiss, LSM 4.2) and then exported to Microsoft Excel for further analysis as previously described (Zha et al., 2006). For any given dendrite, a maximum of the 5 best responding spines were used for analysis. A positive response was called when YFP fluorescence intensity showed obvious increase whereas that of CFP did not.

For quantification of synapse numbers and to minimize variation among neurons from different areas of the hippocampus, we focused our synapse analysis on CA1 pyramidal neurons. We also examined these neurons because previous studies showed changed spine density with changed ASIC1a level in the CA1 region (Zha et al., 2006). Dendritic
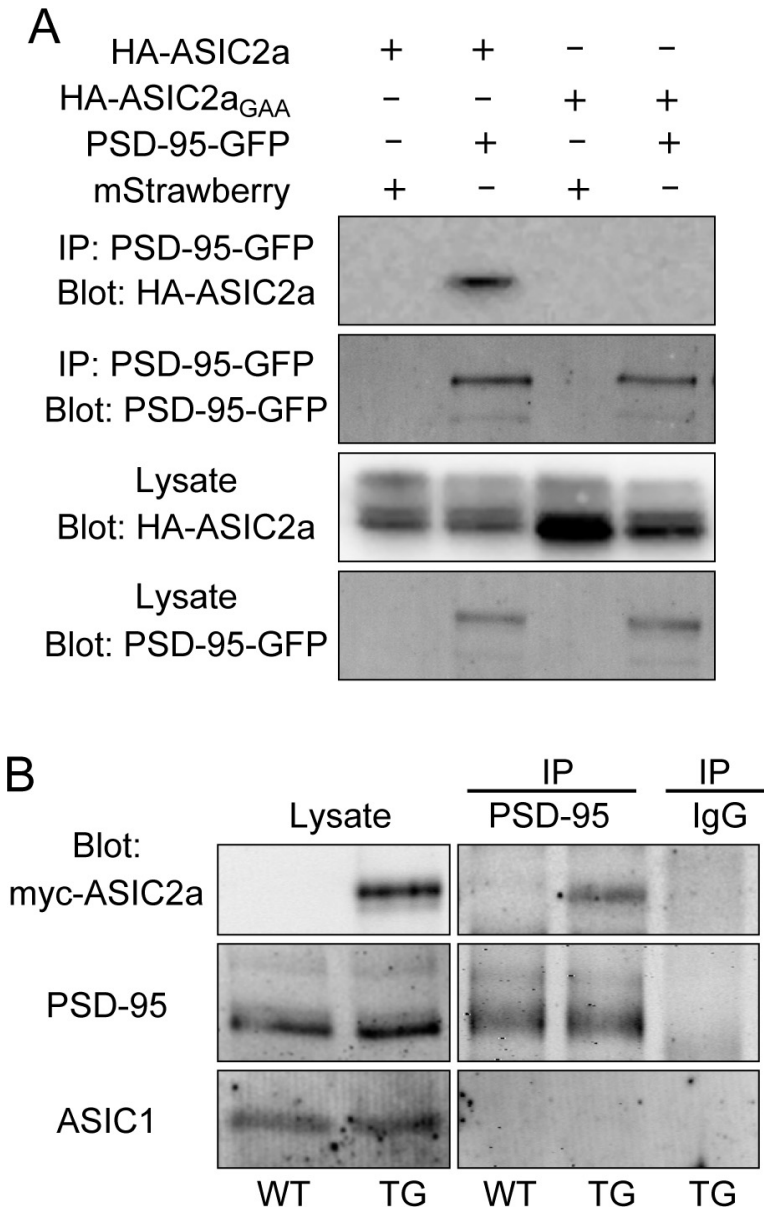

Figure 2. ASIC2a associates with PSD-95 through its PDZ-binding domain. $\boldsymbol{A}, \mathrm{COS}-7$ cells were transfected with HA-ASIC2a or HA-ASIC $2 \mathrm{a}_{\mathrm{GAA}}$ together with PSD-95-GFP or $\mathrm{mStrawberry}$ as indicated. Lysates were immunoprecipitated (IP) with anti-GFP antibody and then immunoblotted for HA. Total lysates from the same cells were also run on SDS-polyacrylamide gels and immunoblotted for HA or GFP to confirm expression. Blots shown are representative from 3 separate experiments. Note that the mutant $A S I C 2 a_{G A A}$ did not interact with PSD-95, showing that the interaction depends on the PDZ-binding motif of ASIC2a. The increase of HA-ASIC2 $\mathrm{a}_{G A A}$ in total lysate was not seen in other experiments. $\boldsymbol{B}$, Coimmunoprecipitation of ASIC2a and PSD-95 from brain. Wild-type and mycASIC2a transgenic mice brain lysates were immunoprecipitated with anti-PSD-95 antibody and immunoblotted for Myc or PSD-95 (the blots for total lysates and immunoprecipitated protein were run on separate gels). Immunoprecipitation with antiPSD-95 but not with control IgG coprecipitated myc-ASIC2a. In contrast, ASIC1 was not coprecipitated with PSD-95. Blots shown are representative of 3 experiments.

segments and synapses were analyzed quantitatively using 3-D image stacks using ImageJ software as described earlier (Zha et al., 2005). Raw data obtained in ImageJ were exported to Microsoft Excel for further analysis.

Statistical analysis. $\left[\mathrm{Ca}^{2+}\right]_{\mathrm{i}}$ results were analyzed using $\chi^{2}$ test. Other data were analyzed using the two tailed Student's $t$ test. Data are reported as mean \pm SEM for the number of samples indicated.

\section{Results}

\section{ASIC2a localizes to synapses}

We examined ASIC2a localization by biolistically transfecting hippocampal slices with HA-tagged ASIC2a and immunostaining with an anti-HA antibody. To facilitate identification of transfected neurons, we coexpressed eGFP. We found ASIC2a immunofluorescence in the soma, in all apical and basal dendrites, and in most dendritic spines (Fig. $1 A, B$ ). We could also detect ASIC2a in axons, although at much lower 
A

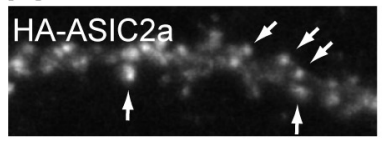

HA-ASIC2a + GFP

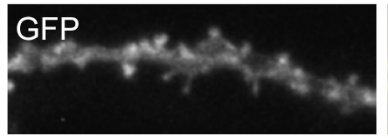

$\mathrm{B}$

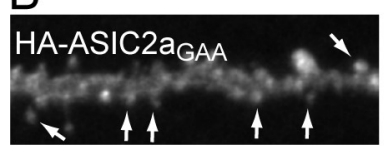

$\mathrm{HA}-\mathrm{ASIC} 2 \mathrm{a}_{\mathrm{GAA}}+\mathrm{GFP}$
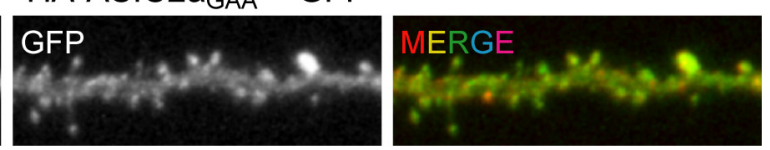

C

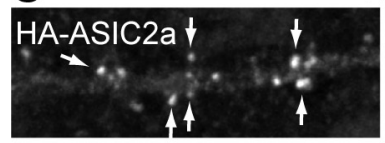

HA-ASIC2a + PSD-95-GFP
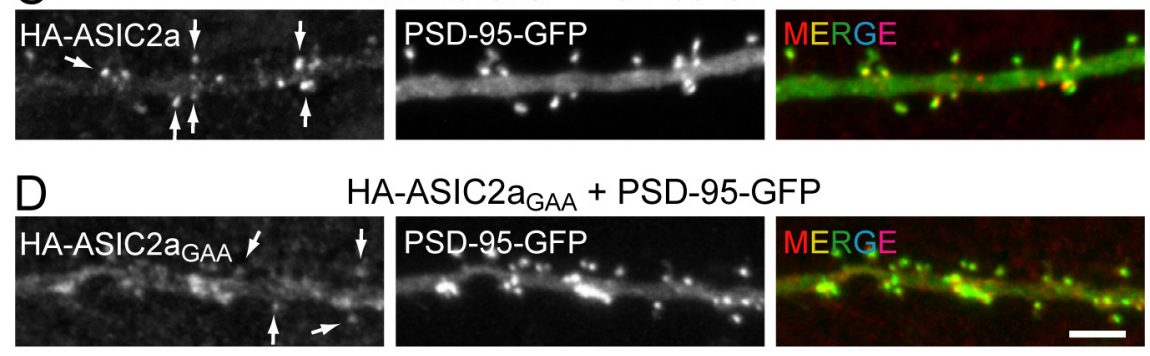

HA-ASIC2a $a_{G A A}+$ PSD-95-GFP
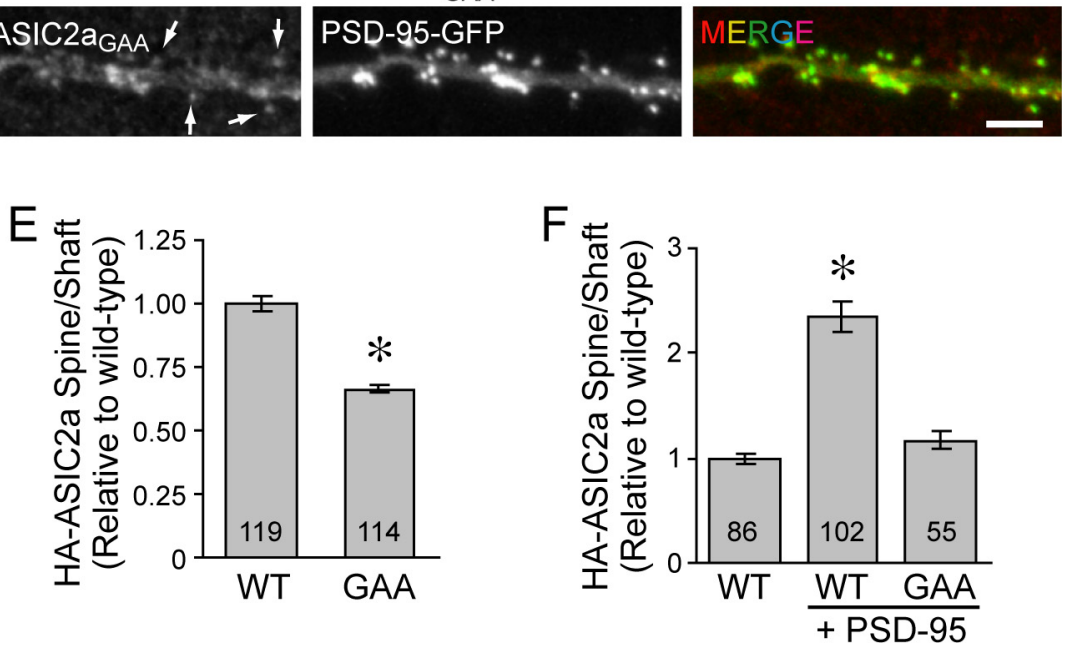

Figure 3. PSD-95 increases ASIC2a in spines. $\boldsymbol{A}-\boldsymbol{D}$, Representative images showing segments of apical dendrites from hippocampal slice neurons transfected with HA-tagged wild-type ASIC2a $(\boldsymbol{A}, \boldsymbol{C})$ or PDZ-binding motif mutated ASIC2a $\mathrm{a}_{\mathrm{GAA}}(\boldsymbol{B}, \boldsymbol{D})$ together with GFP $(\boldsymbol{A}, \boldsymbol{B})$ or PSD-95-GFP $(\boldsymbol{C}, \boldsymbol{D})$. ASIC2a immunofluorescence images are shown on the left, GFP fluorescence images in the middle, and merged images (ASIC2a in red, GFP in green) on the right. Note the difference in ASIC2a immunofluorescence intensity in spine heads (arrows) vs that on adjacent dendritic shaft. Also note that in panel 3D, ASIC2a $\mathrm{GAA}_{\mathrm{GA}}$ was less clustered at most spines than was PSD-95; thus, the merged image shows green spots at these positions. $\boldsymbol{E}$, Quantification of the relative intensity of ASIC2a or ASIC2 $\mathrm{a}_{\mathrm{GAA}}$ in transfected neurons. To eliminate the possible effect of differences in volume, the ratio of ASIC2a in spines vs shafts was normalized against that of GFP. For comparison purpose, the relative ratio of wild-type ASIC2a

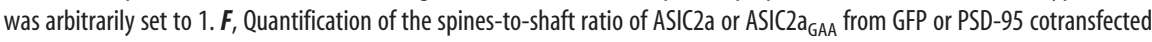
neurons as in $\boldsymbol{A}, \boldsymbol{D}$, and $\boldsymbol{E}\left({ }^{*} p<0.01\right.$, Student's $t$ test; numbers on the bars indicate the total number of spines quantified from 4-7 neurons from 2-3 separate experiments). Scale bar, $4 \mu \mathrm{m}$.

levels (Fig. 1C). In addition, we tested for ASIC2a in the synaptosomal fraction of whole brain of transgenic mice carrying a Myc-tagged ASIC2a. We found Myc-tagged ASIC2a enriched in the synaptosomal fraction (Fig. 1D). These results are consistent with previous reports (Duggan et al., 2002; Jovov et al., 2003; Chai et al., 2007).

PSD-95 interacts with ASIC2a and enhances its localization in dendritic spines

Localization in dendritic spines suggested that ASIC2a might interact with the postsynaptic scaffold. PSD-95 is an important postsynaptic scaffolding protein that interacts with many proteins containing a PDZ-binding motif (Scannevin and Huganir, 2000; Kim and Sheng, 2004). Because the ASIC2 C terminus contains a PDZ-binding motif (Baron et al., 2002b; Duggan et al., 2002; Hruska-Hageman et al., 2002), we asked whether ASIC2a interacts with PSD-95. When expressed in COS-7 cells, PSD-95 coimmunoprecipitated ASIC2a (Fig. 2A). Moreover, mutating the C-terminal three amino acids from Ile-Ala-Cys to residues that do not match a consensus PDZ-binding motif (Doyle et al., 1996; Songyang et al., 1997), Gly-Ala-Ala (ASIC2a $\mathrm{a}_{\mathrm{GAA}}$ ), reduced the interaction. These data indicate that ASIC2a associates with PSD-95, at least in part, through its PDZ-binding motif.

To further test for an association between ASIC2a and PSD-95, we asked whether PSD-95 would coimmunoprecipitate ASIC2 from brain using mycASIC2a transgenic mice (Fig. 2B). AntiPSD-95 antibody coprecipitated mycASIC2a from the whole brain lysate. In contrast, ASIC1a was not precipitated, a result consistent with earlier data showing that ASIC1a did not directly associate with PSD-95 (Hruska-Hageman et al., 2004).

The interaction with PSD-95 suggested that the presence of ASIC2a in dendritic spines might in part depend on PSD-95. Therefore, we examined the localization of ASIC $2 \mathrm{a}_{\mathrm{GAA}}$ and found that the relative amount in dendritic spines $v s$ the dendritic shaft was reduced compared with wildtype ASIC2a (Fig. $3 A, B, E$ ). We also asked whether overexpressing PSD-95 would have the opposite effect. We expressed ASIC2 $a$ and GFP-tagged PSD-95 in neurons and found that they colocalized (Fig. 3C). Moreover, PSD-95 expression enriched ASIC2a immunofluorescence in spines and diminished its presence in dendritic shafts (Fig. 3C,F). Mutating the ASIC2a PDZ-binding motif reduced this effect (Fig. 3D,F). These results suggest that PSD-95 facilitates ASIC2a localization to the postsynaptic sites of excitatory synapses through its interaction with the PDZ-binding motif of ASIC2a.

\section{ASIC2a associates with ASIC1a}

The localization pattern of ASIC2a and its presence in the synaptosomal fraction resembled that of ASIC1a (Wemmie et al., 2002; Zha et al., 2006; Chai et al., 2007). Therefore, we asked whether ASIC2a and ASIC1a colocalize. We cotransfected hippocampal slices with HA-tagged ASIC2a and Flag-tagged ASIC1a. The two ASIC subunits showed almost identical patterns of immunostaining in the dendrites and spines of hippocampal pyramidal neurons (Fig. $4 A, B)$. As in our early study (Zha et al., 2006), we did not detect Flag- or HA-tagged ASIC1a in axons (data not shown). In contrast, when we coexpressed the two subunits, we could detect Flag-ASIC1a and HA-ASIC2a in axons, although at much lower levels (Fig. 4C). These data suggest that coexpressing ASIC2a may lead to a slight increase of ASIC1a in axons. As a control, when we coexpressed Flag-ASIC1a and a different membrane protein, HA-tagged sodium/hydrogen exchanger 1 (NHE1), the two proteins did not substantially colocalize (Fig. 4D). 
To test for an interaction, we immunoprecipitated ASIC1a from mice transgenic for Myc-tagged ASIC2a and found coprecipitation of ASIC2a (Fig. 4E). ASIC2a also coprecipitated ASIC1a. In contrast, neither ASIC2a nor ASIC1a coprecipitated with GluR2, also a synaptic ion channel. The colocalization of ASIC1a and -2a and their physical association are consistent with earlier electrophysiological studies showing that ASIC1a and ASIC2a subunits both contribute to $\mathrm{H}^{+}$-gated current in CNS neurons (Askwith et al., 2004; Chu et al., 2004).

We further studied endogenous ASIC2 in the brain. Among the commercial antibodies we tested (see Materials and Methods), only one recognized a specific ASIC2 band in the wild-type that was absent in the ASIC2 $2^{-/-}$brain (Fig. $5 A$; data not shown). This antibody also recognized several nonspecific bands, therefore precluding immunostaining of endogenous ASIC2. Probing endogenous ASIC2 in lysate and synaptosomes showed an enrichment in the synaptosomal fraction (Fig. 5B), consistent with our data with mice transgenic for mycASIC2a. In addition, we found that immunoprecipitating ASIC1 specifically brought down ASIC2 from wild-type but not ASIC1a $a^{-/-}$mouse brain (Fig. $5 C$ ).

\section{ASIC2a facilitates ASIC1a localization to dendritic spines}

Previous work indicated that ASIC1a does not interact directly with PSD-95 (Hruska-Hageman et al., 2004). However, heterodimerization of ASIC1a with ASIC2a suggested that ASIC2a, through its interaction with PSD-95, might facilitate ASIC1a trafficking to dendritic spines. We tested this hypothesis by comparing HA-ASIC1a localization in slices from wild-type and $A S I C 2^{-/-}$ mice. Consistent with previous results (Zha et al., 2006), ASIC1a was present in both dendritic spines and shafts of wildtype neurons (Fig. 6A). Without ASIC2, the ratio of ASIC1a in spines relative to that in shafts fell (Fig. 6B). As an additional test of the hypothesis, we cotransfected ASIC1a and PSD-95 into either wild-type or $A S I C 2^{-/-}$hippocampal slices. In wild-type neurons, PSD-95 overexpression increased the proportion of ASIC1a in spines vs shafts (Fig. 6C,D). This increase was attenuated in $A S I C 2^{-/-}$neurons.

We looked for a similar effect of ASIC2 on endogenous ASIC1a by assessing ASICla in the synaptosomal fraction of brain lysates. As in previous studies (Wemmie et al., 2002), ASICla was enriched in the synaptosomal fraction of wild-type brain (Fig. $6 E, F$ ). In contrast, enrichment was significantly reduced in $A S I C 2^{-/-}$brain. HA- Flag-

E
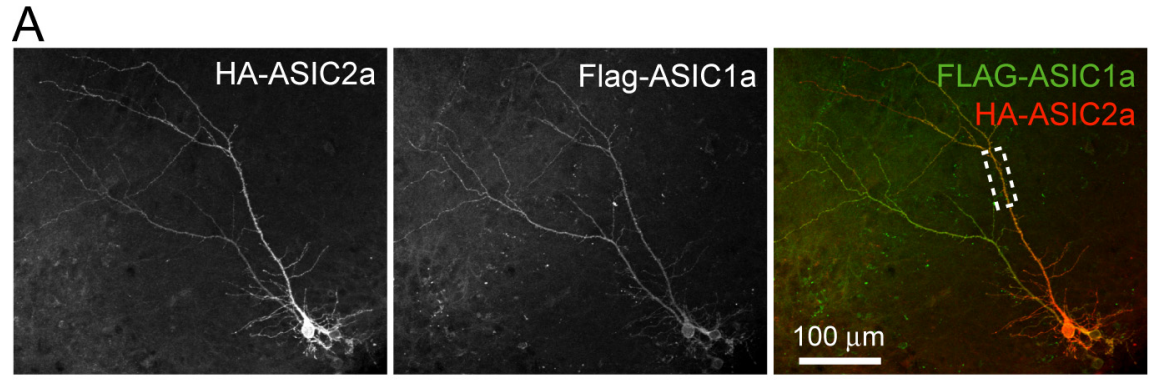

$B$ (boxed region in panel $A$ )

ASIC2a ASIC1a MERGE
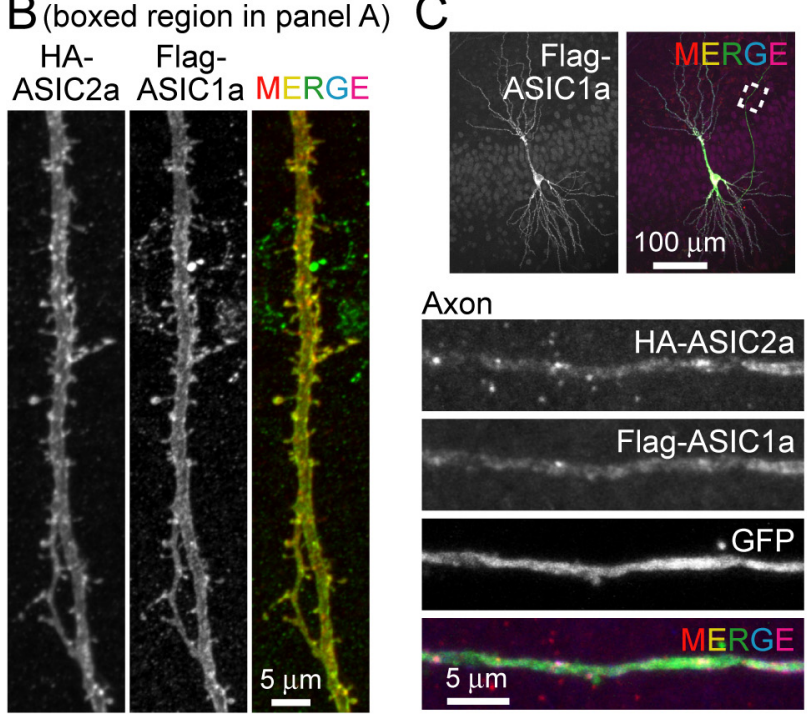

$\mathrm{D}$
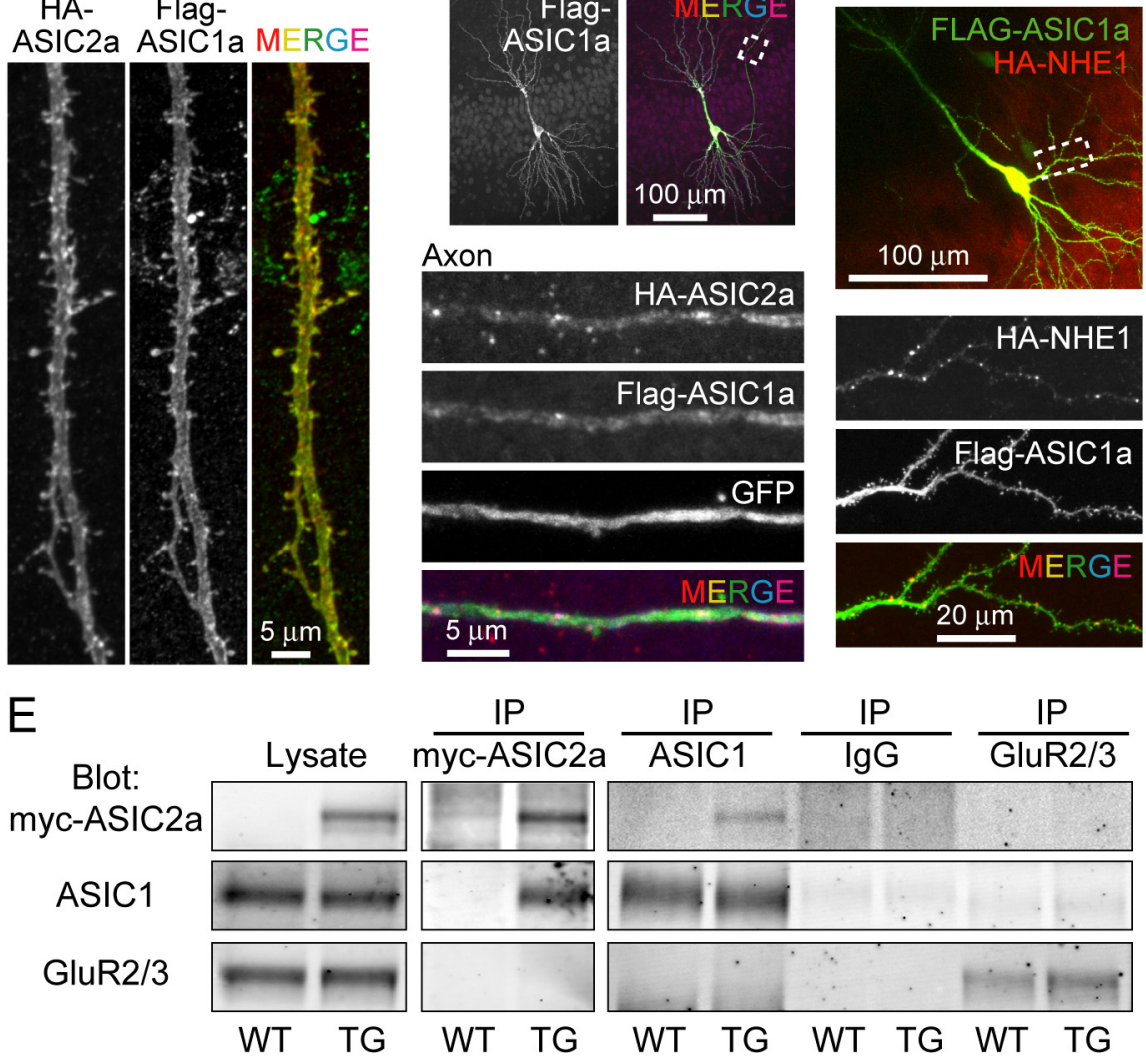

Figure 4. ASIC2a and ASIC1a colocalize in hippocampal slices and interact in vivo. Hippocampal slices were transfected with HA-ASIC2a and Flag-ASIC1a and immunostained with anti-HA and anti-Flag antibodies. A, Low magnification images showing two transfected neurons. $\boldsymbol{B}$, High magnification view of a segment of an apical dendrite (boxed region in $\boldsymbol{A}$ ). Note that ASIC2a and ASIC1a showed almost identical localization at both low and high magnifications. C, ASIC1a was present in axons when coexpressed with ASIC2a. Hippocampal slices were cotransfected with Flag-ASIC1a, HA-ASIC2a and eGFP. Top panels show immunofluorescence image of Flag-ASIC1a on the left and a merged image of Flag-ASIC1a (blue), HA-ASIC2a (red), and GFP (green). Bottom panels show high magnification view of a segment of axon (boxed region in top panel). $D$, Lack of colocalization between ASIC1a and NHE1. Hippocampal slices were transfected with Flag-ASIC1a and HA-NHE1 and immunostained for anti-Flag (green) and anti-HA (red). Top panel shows a low magnification merged image of a transfected neuron. Bottom panels show high magnification view of the boxed dendritic region in the top panel. Note the differences in localization patterns between ASIC 1 a and NHE1. $\boldsymbol{E}$, Representative Western blots from 3 different experiments of coimmunoprecipitation of ASIC1a and Myc-ASIC2a in vivo. Whole brain lysates from wild-type (WT) and Myc-ASIC2a transgenic (TG) mice were immunoprecipitated with anti-Myc, anti-ASIC1a, IgG, and anti-GluR2 antibodies and blotted for Myc-ASIC2a, ASIC1a and GluR2. Immunoprecipitating ASIC1a specifically brought down ASIC2a and vice versa.

Our earlier work showed that activating ASIC1a with an acidic solution elevated $\left[\mathrm{Ca}^{2+}\right]_{i}$ in dendritic spines; reducing and increasing ASIC1a levels decreased and increased, respectively, the percentage of dendritic spines responding to application of a pH 6 solution (Zha et al., 2006). Finding a reduced fraction of ASIC1a at synapses of $A S I C 2^{-1-}$ mice predicted that loss of ASIC2 would also attenuate functional effects of ASIC1a in dendritic spines. We tested the prediction using a 

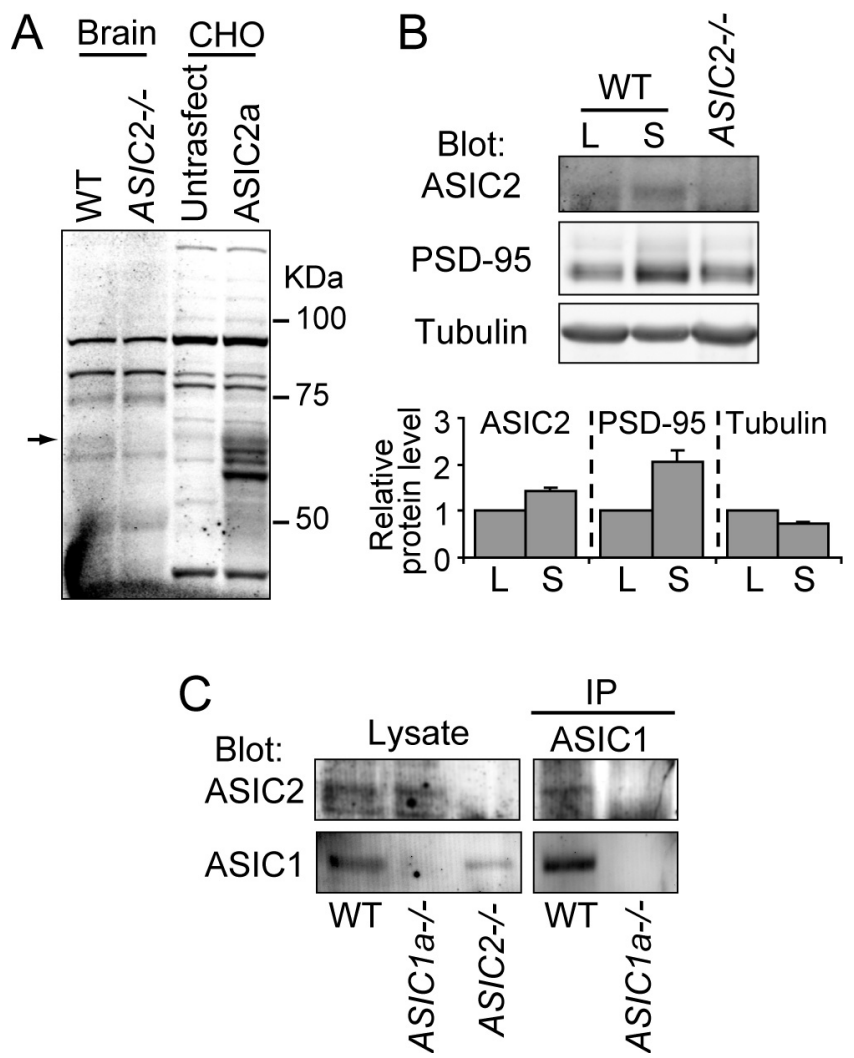

Figure 5. Endogenous ASIC2 enriches in synaptosomes and coimmunoprecipitates with ASIC1. $A$, Blot showing the specificity of the anti-ASIC2 antibody. Arrow indicates ASIC2 band that was absent in the $A S I C 2^{-1-}$ brain (see Materials and Methods). $\boldsymbol{B}$, Western blot of total lysate (L) and synaptosomal fraction (S) of whole brain from wild-type and $A S I C 2^{-/-}$mice, which served as control for antibody specificity. Quantification of relative protein levels is shown in the lower panel. ASIC2 and PSD-95 showed a significant ( $p<0.01$, Student's $t$ test, $N=5$ mice) increase whereas tubulin showed significant $(p<0.001)$ decrease in the synaptosomal fraction. C, Coimmunoprecipitation of endogenous ASIC1 and ASIC2 from mouse brain. Wildtype and $A S I C 1 a^{-1-}$ mice brain lysates were immunoprecipitated with rabbit anti-ASIC1 antibody and blotted with anti-ASIC2 and goat anti-ASIC1. IP with ASIC1 coprecipitated ASIC2 from wild-type but not $A S I C a^{-/-}$brain lysate. The blots shown are representative of 3 similar experiments.

ratiometric $\mathrm{Ca}^{2+}$ reporter (cameleon YC3.60) (Nagai et al., 2004 ) expressed in hippocampal slices. In wild-type slices, a $\mathrm{pH} 6$ solution increased $\left[\mathrm{Ca}^{2+}\right]_{\mathrm{i}}$ in $40 \%$ of dendritic spines (Fig. $7 A, B$ ), consistent with an earlier study (Zha et al., 2006). In contrast, in $A S I C 2^{-/-}$slices only $11 \%$ of dendritic spines responded. These results suggest that ASIC2a binds both PSD-95 and ASIC1a and in so doing facilitates targeting of ASIC1a to synapses. In the absence of ASIC2, there is a decreased response to extracellular acidosis.

\section{ASIC2 is required for normal density of dendritic spines}

ASIC1a modulates the number of dendritic spines; increasing ASIC1a expression increased spine density and reducing ASIC1a decreased it, suggesting altered numbers of synapses (Zha et al., 2006). To learn whether deleting ASIC2 affects dendritic spine density, we expressed PSD-95-GFP to mark and count synapses; although PSD-95 regulates dendrite formation and synapse formation in cultured neurons (El-Husseini et al., 2000; Charych et al., 2006), several studies have shown that transient (48-72 h) PSD-95 expression does not affect synapse or spine density in hippocampal slices (Marrs et al., 2001; Ehrlich and Malinow, 2004; Zha et al., 2005). We found that disrupting the ASIC2 gene reduced the density of synapses (Fig. $8 A, B$ ). The reduction was primarily due to a decrease in spine synapses, with no effect on the density of shaft synapses.

\section{Discussion}

Although widely expressed in central neurons (Price et al., 1996; Waldmann et al., 1996; García-Añoveros et al., 1997), the role of ASIC2 in neuronal function has remained uncertain. Our results show that ASIC2a, through its interaction with PSD-95, is increased in dendritic spines. The association between ASIC2 and ASIC1a subunits also increases the proportion of ASIC1a in synapses. As a result, ASIC1a supports proton-induced elevation of dendritic spine $\left[\mathrm{Ca}^{2+}\right]_{i}$ and consequently the number of synapses.

The findings that ASIC2a homomultimers require a very low $\mathrm{pH}$ for activation ( $a \mathrm{EC}_{50} \mathrm{pH}$ of $\sim 4.8$ ) (Lingueglia et al., 1997; Babinski et al., 2000; Benson et al., 2002), that ASIC2b does not generate $\mathrm{H}^{+}$-gated current (Lingueglia et al., 1997), and that CNS neurons lacking ASIC1a require $\mathrm{pH}$ values $<5.0$ to activate ASIC currents (Askwith et al., 2004) suggested that ASIC2 homomultimers do not play a substantial role in response to physiological reductions in extracellular $\mathrm{pH}$. However, previous studies indicate that ASIC2 subunits can contribute to $\mathrm{H}^{+}$-gated currents when they form heteromultimers with ASIC1a (Baron et al., 2002a; Benson et al., 2002; Askwith et al., 2004). Our current studies further link ASIC2 to ASIC1a and reveal additional mechanisms of interaction. The data show that the two subunits associated in CNS neurons and colocalized at dendritic spines. Moreover, its association with ASIC2 facilitated ASIC1a localization at the postsynaptic membrane. Interestingly, the synaptic scaffolding protein PSD-95 enriched ASIC2a at the synapse, which in turn increased ASIC1a there. Thus, in some respects ASIC2 may serve as a partner that brings or retains ASIC1a in excitatory synapses. The physiological consequences for synaptic function were apparent in $A S I C 2^{-1-}$ neurons as an $\sim 73 \%$ reduction in the percentage of dendritic spines responding to $\mathrm{pH} 6$ solution with a $\left[\mathrm{Ca}^{2+}\right]_{\mathrm{i}}$ elevation. In a previous study in brain slices, we showed that most of this acid-induced $\left[\mathrm{Ca}^{2+}\right]_{\mathrm{i}}$ elevation was due to activation of voltage-gated $\mathrm{Ca}^{2+}$ channels and $\mathrm{Ca}^{2+}$ release from intracellular stores, rather than $\mathrm{Ca}^{2+}$ entry through ASIC1a homomultimers (Zha et al., 2006). Thus, both reducing the number of ASICla subunits at spines and eliminating ASIC1a:ASIC2a heteromultimers would together be expected to attenuate acid-activated $\left[\mathrm{Ca}^{2+}\right]_{\mathrm{i}}$ elevations.

PSD-95 organizes glutamate receptors, adhesion proteins and numerous signaling molecules into a complex that determines synaptic structure and strength (Scannevin and Huganir, 2000; Kim and Sheng, 2004). Our data indicate that ASIC2a is part of that network and that it plays a significant role in neuronal function. Although PSD-95 increased ASIC2a localization in dendritic spines, we cannot say whether it accompanies ASIC2a to the spine or whether it traps ASIC2a there after arrival. Both mechanisms have been observed for PSD-95 and related synaptic scaffolding proteins. For example, AMPA and NMDA receptors trafficking to synapses requires their PDZ binding motif and SAP-97 or SAP-102 (Hayashi et al., 2000; Sans et al., 2001, 2003). Moreover, PSD-95 can stabilize AMPA receptors at synapses (Bats et al., 2007). Likewise, gephrin and homer trap glycine receptors and mGluRs at postsynaptic sites, respectively (Meier et al., 2001; Sergé et al., 2002). In either case, incorporation of ASIC2a 
(and as a result ASIC1a) into the synaptic complex provides the opportunity for the complex to regulate ASIC channel function and for ASIC channels to influence other components of the complex. How does ASIC2 increase ASIC1a in dendritic spines? It seems likely that ASIC2a and ASIC1a will multimerize in the endoplasmic reticulum and then traffic together. Which of the two ASIC2 subunits is primarily responsible for that movement is still uncertain. Although our data show that PSD-95 primarily interacts with ASIC2a, it is possible that PSD-95 or related proteins might weakly interact with ASIC1a, although we were not able to coimmunoprecipitate PSD-95 and ASIC1a in vivo. It is also possible that PSD-95 might indirectly interact with ASIC1a through an ASIC2a-independent mechanism. These speculations are consistent with our observation that ASIC1a was still enriched, although to a lesser extent, in the ASIC2 $2^{-/-}$brain synaptosomes.

Our data indicate that ASIC2a binds PSD-95 through its C-terminal PDZbinding motif. Although that association was key for ASIC2a localization, it is likely not the only interaction involved in ASIC2a localization. For example, PICK1 (Xia et al., 1999) interacts with ASIC1a and -2 (Duggan et al., 2002; Hruska-Hageman et al., 2002) and could also contribute to their presence at the synapse. Both subunits are also reported to interact with AKAP150 and calcineurin (Chai et al., 2007). We also cannot rule out an interaction between ASIC2 and PSD-95 through mechanisms that are either independent of the ASIC2a C terminus or indirectly through another adaptor protein. This speculation would be consistent with the fact that PSD-95 appeared to have a small effect on the localization of the ASIC $2 \mathrm{a}_{\mathrm{GAA}}$ mutant (Fig. 3).

The present results and previous studies suggest that ASIC2 can affect the function of dendritic spines in two ways, by increasing ASICla at synapses and by altering the gating of heteromultimeric ASIC channels. As a result, ASIC2 influences acid-evoked elevations of $\left[\mathrm{Ca}^{2+}\right]_{i}$ in dendritic spines and modulates the number of synapses. Therefore, ASIC2 may also contribute to pathophysiological states where ASIC1a plays a role, including in mouse models of cerebral ischemia, multiple sclerosis, and seizures (Xiong et al., 2004; Yermolaieva et al., 2004; Gao et al., 2005; Friese et al., 2007; Ziemann et al., 2008). Interestingly, one previous report suggested increased ASIC2a expression in neurons surviving ischemia, although the functional consequence of those

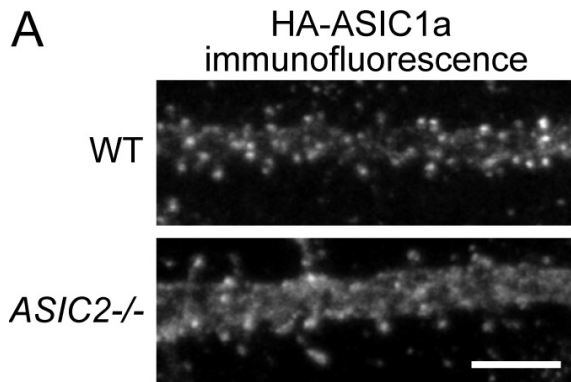

B
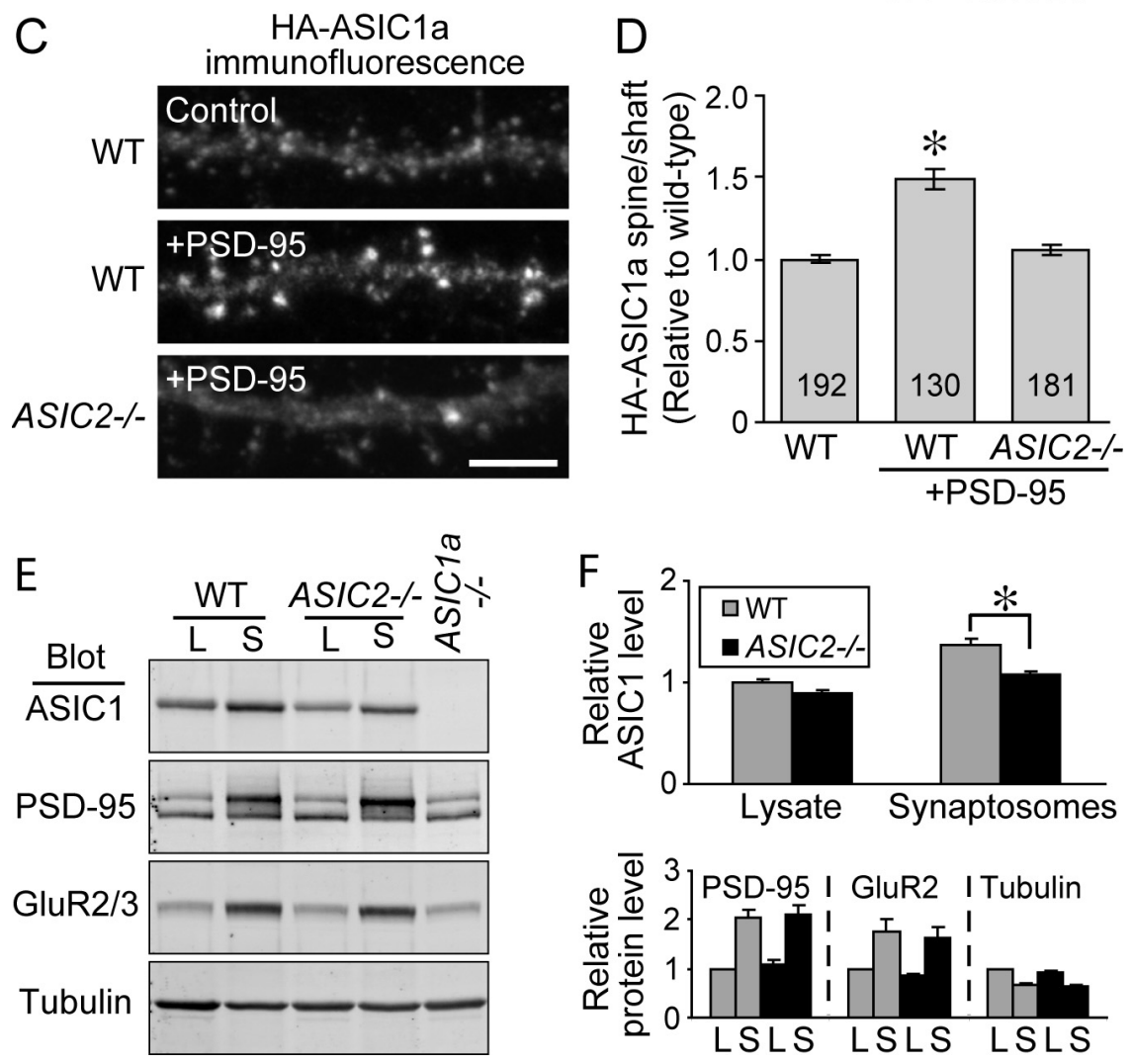

Figure 6. ASIC2a and PSD-95 facilitate ASIC1a targeting to spines. HA-tagged mouse ASIC1a was cotransfected with either GFP or PSD-95-GFP into wild-type or ASIC2 ${ }^{-/-}$hippocampal slices. $\boldsymbol{A}$, Representative images showing ASIC1a immunofluorescence in segments of apical dendrites when cotransfected with GFP. $\boldsymbol{B}$, Quantification of relative spine/ shaft ratios of ASIC1a immunofluorescence. To eliminate potential effects of differences in volume, the ratio of ASIC1a spine to shaft was normalized against that of GFP. For comparison purpose, the ratio of ASIC1a in wild-type neurons was arbitrarily set to 1. C, Representative images showing PSD-95 induced changes in ASIC1a immunofluorescence. D, Quantification of PSD-95 induced changes in ASIC1a spine/shaft ratio. (Numbers on bars indicate the total number of spines quantified from 7-12 neurons from two separate experiments; ${ }^{*} p<0.001$, Student's $t$ test). Scale bar, $5 \mu \mathrm{m}$. $\boldsymbol{E}$, Representative blots of ASIC1a, PSD-95, GluR2, and tubulin in total lysate (L) and synaptosomal fraction (S) of wild-type and $A S I C 2^{-/-}$brains. All 4 antibodies were added together to the same membrane and blotted simultaneously as described in Materials and Methods. Lysate of $A S I C 1 a^{-1-}$ brain served as a negative control for ASIC1a antibody specificity. $\boldsymbol{F}$, Relative protein levels in lysate and synaptosomes from wild-type and $A S I C 2^{-/-}$brains. Top panel shows ASIC1a and bottom panel shows PSD-95, GluR2, and tubulin. Asterisk indicates $p<0.01$ (Student's $t$ test, $N=8$ WT and 8 ASIC $2^{-1-}$ animals from 3 separate experiments). Note that the enrichment of ASIC1a in synaptosomes vs lysates was $37 \pm 6 \%$ in wild-type brain and $20 \pm 4 \%$ in $A S I C 2^{-/-}$brain ( $p<0.05$, Student's $t$ test). In contrast, the enrichment of PSD-95 and GluR2 and the decrease of tubulin in synaptosomes were similar in wild-type and $A S I C 2^{-1-}$ brains.

changes are uncertain (Johnson et al., 2001). Moreover, recent studies suggest genetic associations between the ASIC2 locus and multiple sclerosis, autism and mental retardation (Bernardinelli et al., 2007; Girirajan et al., 2007; Stone et al., 2007). Thus, we speculate that ASIC1a and ASIC2, working in concert, may regulate neuronal function in a variety of disease states. 

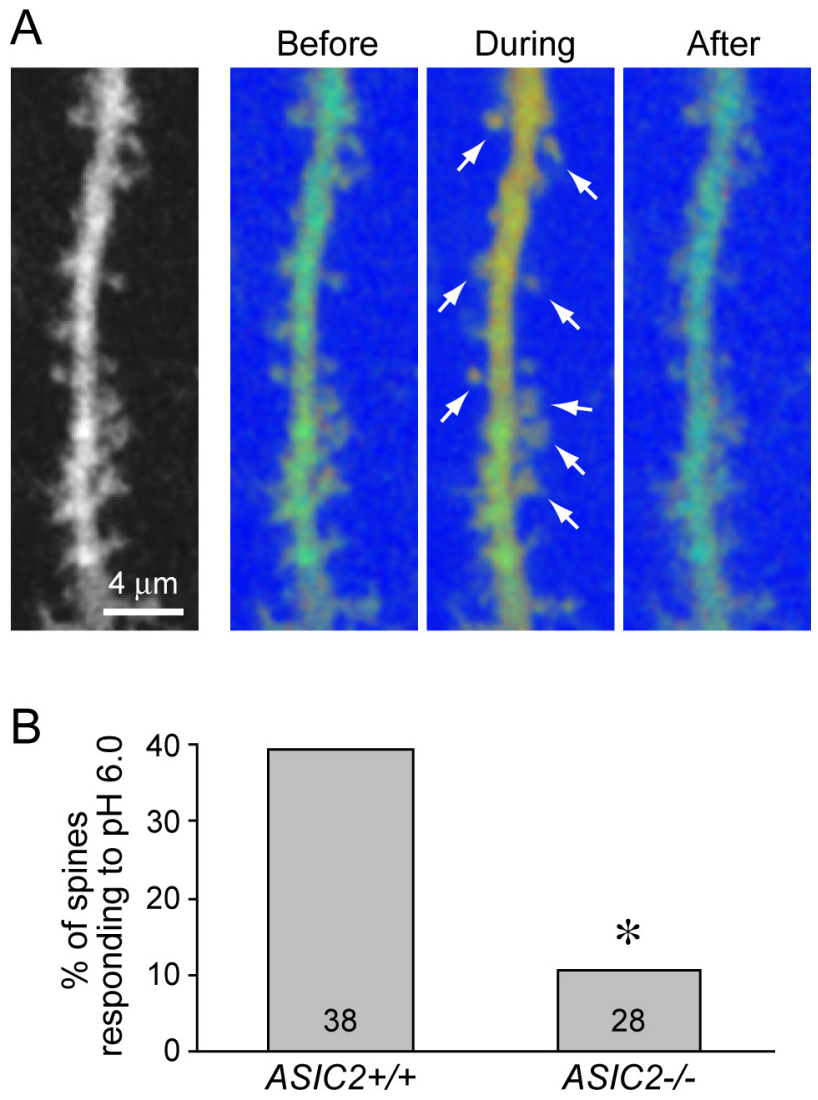

Figure 7. Deleting ASIC2 reduces the percentage of dendritic spines with an acid-evoked $\left[\mathrm{Ca}^{2+}\right]_{\mathrm{i}}$ elevation. $\boldsymbol{A}$, Image on left shows a segment of an apical dendrite from a wild-type neuron transfected with YC3.60. On the right are ratiometric images showing YFP/CFP fluorescence ratio (blue indicates low and red a high ratio) obtained before, during, and after application of pH 6.0 solution. To minimize noise, each ratio image is an average of $15-25$ consecutive scans (4-6s). Note that most dendritic spines and the shaft showed increased FRET during application of acidic solution. $B$, Percentage of all dendritic spines in wild-type and $A S I C 2^{-1-}$ slices that responded to $\mathrm{pH} 6$ solution with an increase in $\left[\mathrm{Ca}^{2+}\right]_{\mathrm{i}}$. Numbers on bars indicate

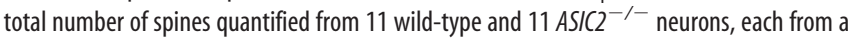
different slice. For any given neuron, a maximum of 5 spines were quantified. Asterisk indicates significant difference $\left(p<0.01\right.$ by $\chi^{2}$ test).

\section{References}

Askwith CC, Wemmie JA, Price MP, Rokhlina T, Welsh MJ (2004) ASIC2 modulates ASIC1 $\mathrm{H}^{+}$-activated currents in hippocampal neurons. J Biol Chem 279:18296-18305.

Babinski K, Catarsi S, Biagini G, Séguéla P (2000) Mammalian ASIC2a and ASIC3 subunits co-assemble into heteromeric proton-gated channels sensitive to $\mathrm{Gd}^{3+}$. J Biol Chem 275:28519-28525.

Baron A, Waldmann R, Lazdunski M (2002a) ASIC-like, proton-activated currents in rat hippocampal neurons. J Physiol 539:485-494.

Baron A, Deval E, Salinas M, Lingueglia E, Voilley N, Lazdunski M (2002b) Protein kinase $\mathrm{C}$ stimulates the acid-sensing ion channel ASIC2a via the PDZ domain-containing protein PICK1. J Biol Chem 277:50463-50468.

Bats C, Groc L, Choquet D (2007) The interaction between Stargazin and PSD-95 regulates AMPA receptor surface trafficking. Neuron 53:719-734.

Benson CJ, Xie J, Wemmie JA, Price MP, Henss JM, Welsh MJ, Snyder PM (2002) Heteromultimerics of DEG/ENaC subunits form $\mathrm{H}^{+}$-gated channels in mouse sensory neurons. Proc Natl Acad Sci USA 99:2338-2343.

Berdiev BK, Xia J, McLean LA, Markert JM, Gillespie GY, Mapstone TB, Naren AP, Jovov B, Bubien JK, Ji HL, Fuller CM, Kirk KL, Benos DJ (2003) Acid-sensing ion channels in malignant gliomas. J Biol Chem 278:15023-15034.

Bernardinelli L, Murgia SB, Bitti PP, Foco L, Ferrai R, Musu L, Prokopenko I, Pastorino R, Saddi V, Ticca A, Piras ML, Cox DR, Berzuini C (2007)
A
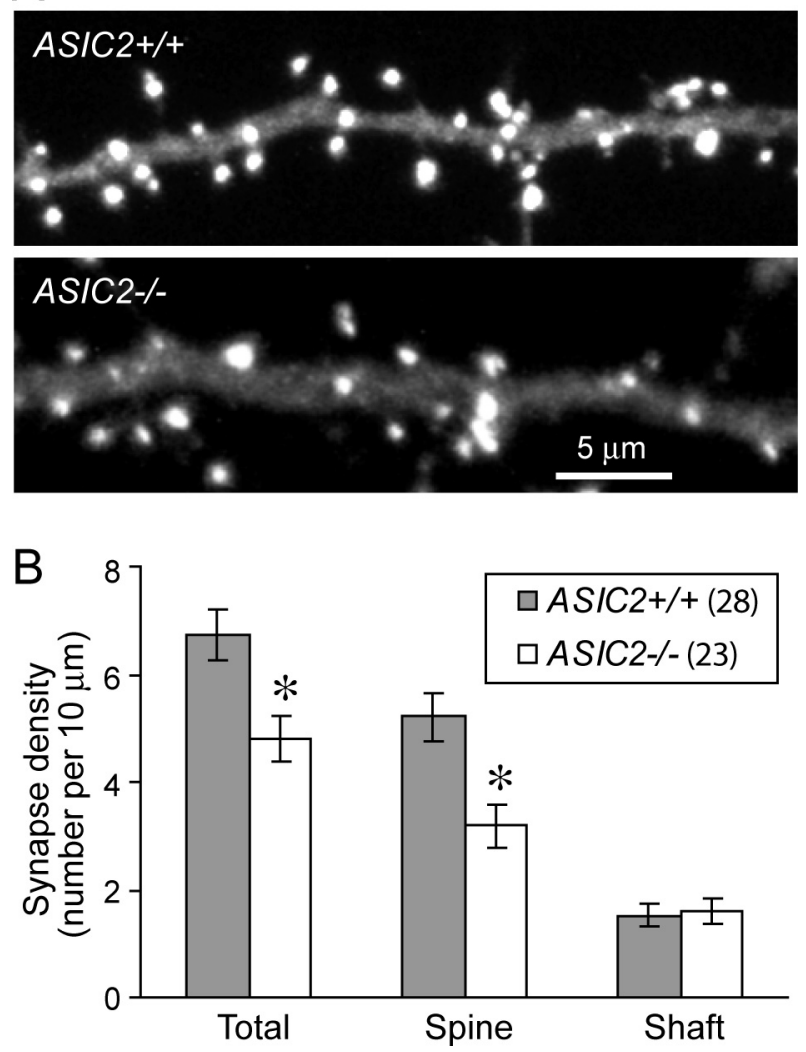

Figure 8. Eliminating ASIC2 decreases the density of dendritic spines. A, GFP fluorescence images of a segment of apical dendrites from wild-type and $A S I C 2^{-/-}$slice neurons transfected with PSD-95-GFP. Note that PSD-95-GFP that is clustered in spines and along the dendritic shaft indicates the postsynaptic site of excitatory synapses. $\boldsymbol{B}$, Quantification of synapse density reveals significant differences between control and $A S I C 2^{-1-}$ in total and spine synapses. Numbers in parentheses indicate total number of neurons quantified from 3 different experiments ( ${ }^{*} p<0.01$ compared with wild-type).

Association between the ACCN1 gene and multiple sclerosis in Central East Sardinia. PLoS ONE 2:e480.

Chai S, Li M, Lan J, Xiong ZG, Saugstad JA, Simon RP (2007) A kinaseanchoring protein 150 and calcineurin are involved in regulation of acidsensing ion channels ASIC1a and ASIC2a. J Biol Chem 282:22668-22677.

Charych EI, Akum BF, Goldberg JS, Jörnsten RJ, Rongo C, Zheng JQ, Firestein BL (2006) Activity-independent regulation of dendrite patterning by postsynaptic density protein PSD-95. J Neurosci 26:10164-10176.

Chu XP, Wemmie JA, Wang WZ, Zhu XM, Saugstad JA, Price MP, Simon RP, Xiong ZG (2004) Subunit-dependent high-affinity zinc inhibition of acid-sensing ion channels. J Neurosci 24:8678-8689.

Coryell MW, Ziemann AE, Westmoreland PJ, Haenfler JM, Kurjakovic Z, Zha XM, Price M, Schnizler MK, Wemmie JA (2007) Targeting ASIC1a reduces innate fear and alters neuronal activity in the fear circuit. Biol Psychiatry 62:1140-1148.

Doyle DA, Lee A, Lewis J, Kim E, Sheng M, MacKinnon R (1996) Crystal structures of a complexed and peptide-free membrane protein-binding domain: molecular basis of peptide recognition by PDZ. Cell 85:1067-1076.

Duggan A, Garcia-Anoveros J, Corey DP (2002) The PDZ domain protein PICK1 and the sodium channel $\mathrm{BNaCl}$ interact and localize at mechanosensory terminals of dorsal root ganglion neurons and dendrites of central neurons. J Biol Chem 277:5203-5208.

Ehrlich I, Malinow R (2004) Postsynaptic density 95 controls AMPA receptor incorporation during long-term potentiation and experience-driven synaptic plasticity. J Neurosci 24:916-927.

El-Husseini AE, Schnell E, Chetkovich DM, Nicoll RA, Bredt DS (2000) PSD-95 involvement in maturation of excitatory synapses. Science 290:1364-1368.

Ettaiche M, Guy N, Hofman P, Lazdunski M, Waldmann R (2004) Acid- 
sensing ion channel 2 is important for retinal function and protects against light-induced retinal degeneration. J Neurosci 24:1005-1012.

Friese MA, Craner MJ, Etzensperger R, Vergo S, Wemmie JA, Welsh MJ, Vincent A, Fugger L (2007) Acid-sensing ion channel-1 contributes to axonal degeneration in autoimmune inflammation of the central nervous system. Nat Med 13:1483-1489.

Gao J, Wu LJ, Xu L, Xu TL (2004) Properties of the proton-evoked currents and their modulation by $\mathrm{Ca}^{2+}$ and $\mathrm{Zn}^{2+}$ in the acutely dissociated hippocampus CA1 neurons. Brain Res 1017:197-207.

Gao J, Duan B, Wang DG, Deng XH, Zhang GY, Xu L, Xu TL (2005) Coupling between NMDA receptor and acid-sensing ion channel contributes to ischemic neuronal death. Neuron 48:635-646.

García-Añoveros J, Derfler B, Neville-Golden J, Hyman BT, Corey DP (1997) $\mathrm{BNaC} 1$ and $\mathrm{BNaC} 2$ constitute a new family of human neuronal sodium channels related to degenerins and epithelial sodium channels. Proc Natl Acad Sci U S A 94:1459-1464.

Girirajan S, Williams SR, Garbern JY, Nowak N, Hatchwell E, Elsea SH (2007) $17 \mathrm{p} 11.2 \mathrm{p} 12$ triplication and $\operatorname{del}(17) \mathrm{q} 11.2 \mathrm{q} 12$ in a severely affected child with dup(17)p11.2p12 syndrome. Clin Genet 72:47-58.

Hayashi Y, Shi SH, Esteban JA, Piccini A, Poncer JC, Malinow R (2000) Driving AMPA receptors into synapses by LTP and CaMKII: requirement for GluR1 and PDZ domain interaction. Science 287:2262-2267.

Hruska-Hageman AM, Wemmie JA, Price MP, Welsh MJ (2002) Interaction of the synaptic protein PICK1 (protein interacting with $\mathrm{C}$ kinase 1) with the non-voltage-gated sodium channels BNC1 (brain $\mathrm{Na}^{+}$channel 1) and ASIC (acid-sensing ion channel). Biochem J 361:443-450.

Hruska-Hageman AM, Benson CJ, Leonard AS, Price MP, Welsh MJ (2004) PSD-95 and Lin-7b interact with acid-sensing ion channel-3 and have opposite effects on H+-gated current. J Biol Chem 279:46962-46968.

Johnson MB, Jin K, Minami M, Chen D, Simon RP (2001) Global ischemia induces expression of acid-sensing ion channel 2a in rat brain. J Cereb Blood Flow Metab 21:734-740.

Jovov B, Tousson A, McMahon LL, Benos DJ (2003) Immunolocalization of the acid-sensing ion channel 2a in the rat cerebellum. Histochem Cell Biol 119:437-446.

Kim E, Sheng M (2004) PDZ domain proteins of synapses. Nat Rev Neurosci 5:771-781.

Lingueglia E, de Weille JR, Bassilana F, Heurteaux C, Sakai H, Waldmann R, Lazdunski M (1997) A modulatory subunit of acid sensing ion channels in brain and dorsal root ganglion cells. J Biol Chem 272:29778-29783.

Marrs GS, Green SH, Dailey ME (2001) Rapid formation and remodeling of postsynaptic densities in developing dendrites. Nat Neurosci 4:1006-1013.

Matsuda T, Cooper JR (1981) Thiamine as an integral component of brain synaptosomal membranes. Proc Natl Acad Sci U S A 78:5886-5889.

Meier J, Vannier C, Sergé A, Triller A, Choquet D (2001) Fast and reversible trapping of surface glycine receptors by gephyrin. Nat Neurosci 4:253-260.

Nagai T, Yamada S, Tominaga T, Ichikawa M, Miyawaki A (2004) Expanded dynamic range of fluorescent indicators for $\mathrm{Ca}(2+)$ by circularly permuted yellow fluorescent proteins. Proc Natl Acad Sci U S A 101:10554-10559.

Price MP, Snyder PM, Welsh MJ (1996) Cloning and expression of a novel human brain $\mathrm{Na}^{+}$channel. J Biol Chem 271:7879-7882.

Sans N, Racca C, Petralia RS, Wang YX, McCallum J, Wenthold RJ (2001) Synapse-associated protein 97 selectively associates with a subset of
AMPA receptors early in their biosynthetic pathway. J Neurosci 21:7506-7516.

Sans N, Prybylowski K, Petralia RS, Chang K, Wang YX, Racca C, Vicini S, Wenthold RJ (2003) NMDA receptor trafficking through an interaction between PDZ proteins and the exocyst complex. Nat Cell Biol 5:520-530.

Scannevin RH, Huganir RL (2000) Postsynaptic organization and regulation of excitatory synapses. Nat Rev Neurosci 1:133-141.

Sergé A, Fourgeaud L, Hémar A, Choquet D (2002) Receptor activation and homer differentially control the lateral mobility of metabotropic glutamate receptor 5 in the neuronal membrane. J Neurosci 22:3910-3920.

Songyang Z, Fanning AS, Fu C, Xu J, Marfatia SM, Chishti AH, Crompton A, Chan AC, Anderson JM, Cantley LC (1997) Recognition of unique carboxyl-terminal motifs by distinct PDZ domains. Science 275:73-77.

Stone JL, Merriman B, Cantor RM, Geschwind DH, Nelson SF (2007) High density SNP association study of a major autism linkage region on chromosome 17. Hum Mol Genet 16:704-715.

Waldmann R, Champigny G, Voilley N, Lauritzen I, Lazdunski M (1996) The mammalian degenerin MDEG, an amiloride-sensitive cation channel activated by mutations causing neurodegeneration in Caenorhabditis elegans. J Biol Chem 271:10433-10436.

Waldmann R, Champigny G, Bassilana F, Heurteaux C, Lazdunski M (1997) A proton-gated cation channel involved in acid-sensing. Nature 386:173-177.

Wemmie J, Coryell M, Askwith C, Lamani E, Leonard S, Sigmund C, Welsh M (2004) Overexpression of acid-sensing ion channel la in transgenic mice increases fear-related behavior. Proc Natl Acad Sci U S A 101:3621-3626.

Wemmie JA, Chen J, Askwith CC, Hruska-Hageman AM, Price MP, Nolan BC, Yoder PG, Lamani E, Hoshi T, Freeman JH Jr, Welsh MJ (2002) The acid-activated ion channel ASIC contributes to synaptic plasticity, learning, and memory. Neuron 34:463-477.

Wemmie JA, Askwith CC, Lamani E, Cassell MD, Freeman JHJ, Welsh MJ (2003) Acid-sensing ion channel 1 is localized in brain regions with high synaptic density and contributes to fear conditioning. J Neurosci 23:5496-5502.

Xia J, Zhang X, Staudinger J, Huganir RL (1999) Clustering of AMPA receptors by the synaptic PDZ domain-containing protein PICK1. Neuron 22:179-187.

Xiong ZG, Zhu XM, Chu XP, Minami M, Hey J, Wei WL, MacDonald JF, Wemmie JA, Price MP, Welsh MJ, Simon RP (2004) Neuroprotection in ischemia: blocking calcium-permeable acid-sensing ion channels. Cell 118:687-698.

Yermolaieva O, Leonard AS, Schnizler MK, Abboud FM, Welsh MJ (2004) Extracellular acidosis increases neuronal cell calcium by activating acidsensing ion channel 1a. Proc Natl Acad Sci U S A 101:6752-6757.

Zha XM, Green SH, Dailey ME (2005) Regulation of hippocampal synapse remodeling by epileptiform activity. Mol Cell Neurosci 29:494-506.

Zha XM, Wemmie JA, Green SH, Welsh MJ (2006) ASICla is a postsynaptic proton receptor that influences the density of dendritic spines. Proc Natl Acad Sci U S A 103:16556-16561.

Zha XM, Wang R, Collier DM, Snyder PM, Wemmie JA, Welsh MJ (2009) Oxidant regulated inter-subunit disulfide bond formation between ASIC1a subunits. Proc Natl Acad Sci U S A 106:3573-3578.

Ziemann AE, Schnizler MK, Albert GW, Severson MA, Howard MA 3rd, Welsh MJ, Wemmie JA (2008) Seizure termination by acidosis depends on ASICla. Nat Neurosci 11:816-822. 